\section{(6) OPEN ACCESS}

\title{
Toll-like receptor-mediated, enhanced production of profibrotic TIMP-1 in monocytes from patients with systemic sclerosis: role of serum factors
}

\author{
Marzena Ciechomska, ${ }^{1}$ Christiaan A Huigens, ${ }^{1}$ Thomas Hügle, ${ }^{2}$ Tess Stanly, ${ }^{1}$ \\ Andreas Gessner, ${ }^{1}$ Bridget Griffiths, ${ }^{3}$ Timothy R D J Radstake, ${ }^{4}$ Sophie Hambleton, ${ }^{1}$ \\ Steven O'Reilly, ${ }^{1}$ Jacob M van Laar ${ }^{1}$
}

Handling editor Tore K Kvien

- Additional data are published online only. To view these files please visit the journal online (http://dx.doi.org/ 10.1136/annrheumdis-2012 201958)

${ }^{1}$ Musculoskeletal Research Group, Institute of Cellular Medicine, Newcastle University, Newcastle upon Tyne, UK

${ }^{2}$ Department of Rheumatology, University Hospital Basel, Basel, Switzerland

${ }^{3}$ Department of Rheumatology, Freeman Hospital, Newcastle upon Tyne, UK

${ }^{4}$ Department of Rheumatology and Clinical Immunology,

University Medical

Centre Utrecht, Utrecht,

The Netherlands

\section{Correspondence to}

Professor Jacob M van Laar,

Musculoskeletal Research

Group, Institute of Cellular

Medicine, Newcastle

University, 4th Floor, Catherine Cookson Building, The Medical School, Framlington Place, Newcastle upon Tyne NE2 4HH, UK;

j.m.van-laar@ncl.ac.uk

$\mathrm{MC}$ and CAH contributed equally.

Received 4 May 2012

Revised 23 October 2012

Accepted 18 November 2012

Published Online First

6 December 2012

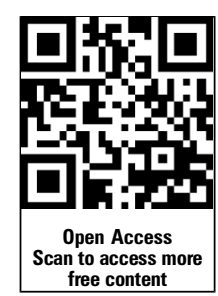

\section{ABSTRACT}

Objectives To investigate whether monocytes contribute to matrix deposition in systemic sclerosis (SSc) by production of tissue-inhibitor of metalloproteinase-1 (TIMP-1).

Methods Matrix metalloproteinase-1 (MMP-1) and TIMP-1 expression and secretion were measured by qRT-PCR and ELISA in circulating monocytes from patients with SSc, patients with rheumatoid arthritis (RA) and healthy controls $(\mathrm{HC})$ and in healthy monocytes cultured in the presence of SSc or HC serum samples. Production of TIMP-1 was determined in response to a panel of Toll-like receptor (TLR) agonists and MyD88 inhibitory peptide. The functional effect of conditioned media from SSc and HC serum samples or TLR8stimulated monocytes was studied in an MMP-1 activity assay.

Results TIMP-1 production by monocytes was upregulated in patients with SSc compared with patients with RA and $\mathrm{HC}$. Incubation of HC monocytes with SSc serum samples resulted in functionally active TIMP-1 production. However, pretreatment with MyD88 inhibitor, but not control peptide, decreased TIMP-1 secretion. TIMP-1 production was significantly stronger when SSc and $\mathrm{HC}$ monocytes were stimulated with TLR8 (ssRNA) agonist, but the response was more pronounced in SSc monocytes. TIMP-1 production after TLR stimulation was also strongly reduced in the presence of MyD88 inhibitory peptide or in the monocytes isolated from a patient with a genetic TLR signalling defect. MMP-1 activity was significantly inhibited in media from serum samples or TLR8-stimulated monocytes indicative of functional TIMP activity.

Conclusions This study demonstrates profibrotic properties of circulating monocytes from patients with SSc and a key role for TLR signalling, particularly TLR8, in TIMP-1 secretion and matrix remodelling.

\section{INTRODUCTION}

Systemic sclerosis (SSc) is a rare autoimmune connective tissue disease characterised by vasculopathy and fibrosis of the skin and inner organs. Low-grade inflammation with tissue infiltration by mononuclear cells plays an important role in fibrogenesis. ${ }^{1}$ Fibrosis in SSc is characterised by extensive accumulation of extracellular matrix (ECM), including collagen. In addition to increased secretion of ECM components, impaired breakdown of the ECM can also contribute to fibrosis. ${ }^{2}$ ECM breakdown is mainly mediated by matrix metalloproteinases (MMPs), a family of endopeptidases capable of degrading all matrix components. ${ }^{3}$ Tissue inhibitors of metalloproteinase (TIMPs) selectively inhibit these peptidases, thus inhibiting ECM breakdown. ${ }^{3} 4$ The TIMP family consists of four members; of which, TIMP-1 is a key enzyme as it can inhibit most MMPs. ${ }^{3}$ Several studies have reported increased serum concentrations of TIMP-1 and increases in the ratio of TIMP/MMP in both tissue and blood of patients with SSc. ${ }^{5-8}$ Wound healing studies have shown that TIMP-1 expressed by inflammatory cells such as monocytes or macrophages is important in tissue remodelling. Studies have identified circulating monocytes as key inflammatory cells in SSc, but which factor drives TIMP-1 is still undefined. ${ }^{10} 11$

Toll-like receptors (TLRs) are integral components of the innate immune system that recognise pathogen-encoded TLR ligands, including viral and bacterial fragments. ${ }^{12}$ Most TLRs function through the myeloid differentiation protein 88 (MyD88) adapter protein. Homodimerisation of MyD88 is a critical step in the downstream signalling process, which allows the recruitment and activation of the interleukin 1 receptor (IL-1R)-associated kinase (IRAK), consequently leading to the expression of proinflammatory and profibrotic cytokines, chemokines, but also collagen. ${ }^{13} 14$

MyD88-deficient rats and mice are protected from fibrosis, pathological inflammation and cardiac hypertrophy, implying a crucial role for MyD88 in fibrogenesis. ${ }^{15}$ TLR-mediated activation can also be induced by recognition of self-reactive nucleic acids that bind to intracellular TLR7, 8 or 9 , consequently initiating the breakdown of tolerance and promoting the development of autoimmune disease. ${ }^{16} 17$ It was previously reported that autoantigens can bind to IgG and form immune complexes (ICs) that subsequently induce interferon $\alpha$ (IFN $\alpha$ ) production by plasmacytoid dendritic cells ( $\mathrm{pDCs}$ ) in systemic lupus erythematosus (SLE) and Sjögren's syndrome. ${ }^{18-21}$ However, whether a similar mechanism is at play in SSc is unknown. Here we show that SSc monocytes stimulated with TLR agonists, in particular TLR8 (ssRNA), produce TIMP-1, leading to a shift in the balance between MMP-1 and TIMP-1 and altered 
matrix remodelling in vitro. Furthermore, serum samples from patients with SSc are a source of MyD88-dependent TLR agonists driving TIMP-1 production and therefore promoting fibrosis development. Our findings point to a critical role of circulating monocytes in fibrogenesis in SSc.

\section{MATERIALS AND METHODS \\ Patients and controls}

Twenty-three patients with SSc, 29 healthy controls (HC) and 21 patients with active rheumatoid arthritis (RA) were included in the study. All patients with SSc fulfilled the American College of Rheumatology criteria according to LeRoy. Cells from an IRAK4-deficient patient were also used in this study, characterised by impairment of neutrophil CD62L shedding and absent cytokine responses to TLR ligands, typical of patients with autosomal recessive deficiency of IRAK4 or MyD88. ${ }^{22} 23$

\section{Sample collection and cell purification}

Blood was collected in EDTA-coated tubes from HC, patients with SSc or patients with RA during standard outpatient procedures and processed within $4 \mathrm{~h}$ of collection. Peripheral blood mononuclear cells were separated from whole blood by Ficoll-Hypaque density gradient centrifugation (Axis-shield). CD14 monocytes were isolated from peripheral blood mononuclear cells, according to the manufacturer's protocol, with the CD14 MACS beads isolation kit (Miltenyi-Biotec, Bergisch Gladbach, Germany). Purified monocytes were removed from the column and tested for purity by flow cytometry (showing $>95 \%$ purity for CD14+CD3- cells). Serum samples were collected in 'serum separation tubes' (Grainer, Vacuette), aliquoted and frozen at $-20^{\circ} \mathrm{C}$. Some $\mathrm{HC}$ and SSc serum samples were also pretreated for $4 \mathrm{~h}$ with 4 units of benzonase (Sigma-E1014) per $1 \mu \mathrm{l}$ of the serum samples at $37^{\circ} \mathrm{C}$.

\section{In vitro monocyte cultures}

CD14 monocytes from HC or patients with SSc were seeded in 48-well Costar plates at a concentration of $1 \times 10^{6}$ cells $/ \mathrm{ml}$ and cultured for 4-24h in 300-600 $\mu \mathrm{l}$ of Dulbecco's modified Eagle's medium (DMEM; Invitrogen) supplemented with penicillin $(100 \mathrm{U} / \mathrm{ml})$, streptomycin $(100 \mu \mathrm{g} / \mathrm{ml})$, L-glutamine (2 mM) (all Sigma) and 10\% serum from patients with SSc, patients with $\mathrm{RA}$ or $\mathrm{HC}$ at $37^{\circ} \mathrm{C}$ in $5 \% \mathrm{CO}_{2}$.

\section{Stimulation and immunohistochemistry of TLR agonists}

A human TLR1-9 Agonists Kit was purchased from InvivoGen, resuspended in endotoxin-free water and stored in sterile conditions at $-20^{\circ} \mathrm{C}$. The set of TLR 1-9 agonists was used at the following final concentrations: TLR1/2 (Pam3CSK4) $(1 \mu \mathrm{g} / \mathrm{ml})$, TLR2 (HKLM) $\left(10^{8}\right.$ cells/ml), TLR3 (poly(I:C) $(1 \mu \mathrm{g} / \mathrm{ml})$, TLR4 (lipopolysaccharide) $(1 \mu \mathrm{g} / \mathrm{ml})$, TLR5 (flagellin) $(1 \mu \mathrm{g} / \mathrm{ml})$, TLR6/2 (FSL-1) $(1 \mu \mathrm{g} / \mathrm{ml})$, TLR7 (imiquimod) $(1 \mu \mathrm{g} / \mathrm{ml})$, TLR8 (ssRNA40) $(1 \mu \mathrm{g} / \mathrm{ml})$, TLR9 (ODN2006 (type B)) (5 mM). A MyD88 Homodimerisation Inhibitory Peptide Set (Imgenex, IMG-2005-5) was resuspended in phosphate-buffered saline and used at $50 \mu \mathrm{M}$, as recommended by the manufacturer. A paraffin-embedded skin section from a patient with SSc was stained with goat anti-human CD14 (Abcam, ab45870) and mouse anti-human TIMP-1 (Abcam, ab1827) primary antibodies overnight at $4^{\circ} \mathrm{C}$ and with secondary antibodies (Invitrogen, anti-goat-Alexa-Fluor488 anti-mouse-Alexa-Fluor546, respectively). The skin section was further analysed by confocal microscopy.
Semiquantitative MMP-1 and TIMP-1 gene expression analysis

RNA from freshly isolated monocytes was obtained using the RNA mini kit (Qiagen), according to the manufacturer's protocol. RNA (200-750 ng) was treated with DNAse and reverse transcribed to cDNA with the use of random hexamers and the Moloney murine leukaemia virus reverse transcriptase enzyme (Invitrogen), according to the manufacturer's protocol. cDNA (20 ng), forward and reverse primer and probe in Taq ready mastermix was used for TIMP-1 expression analyses (see online supplementary table S1). MMP-1 expression was analysed using SYBR Green mix. Samples were analysed in triplicate and normalised to the $18 \mathrm{~S}$ housekeeping gene using the AB7500 (Applied Biosystems) qRT-PCR machine and programme. Expression levels relative to the average healthy control (arbitrarily set at 1) were calculated using the following equation: $\left(2^{\wedge} \text { Delta Delta CT }\right)^{-1}$ all normalised to $18 \mathrm{~S}$ housekeeping gene. Primers, probes and Taq ready mastermix were used according to the manufacturer's protocol (Sigma-Aldrich, St Louis, MO, USA).

\section{TIMP and MMP ELISA}

TIMP-1, TIMP-2, MMP-1 (R\&D Systems) and MMP-13 (in-house kit) protein concentrations in culture supernatants and serum samples were measured by ELISA, according to the manufacturer's protocol. Signal development was performed using horseradish peroxidase/streptavidin and o-phenylenediamine dihydrochloride substrate (Sigma-Aldrich) at room temperature. Fluorescence was measured with a plate reader (Tecan, Sunrise). Samples were run in duplicate and serial dilution was performed to fall within the detection limits of the assay $(0-100 \mathrm{ng} / \mathrm{ml})$.

\section{Real-time fluorescence-based MMP-1 activity matrix assay}

The functional effect of increased expression and secretion of TIMPs in monocytes on MMP activity was studied by a real-time fluorescence based MMP activity assay. ${ }^{21}$ Human proMMP-1 (Calbiotech) was activated by 4-aminophenylmercuric acetate (0.675 mM) (Sigma) in 0.1 M Tris, pH 7.5, $0.1 \mathrm{M} \mathrm{NaCl}, 10 \mathrm{mM}$ $\mathrm{CaCl}_{2}, 0.05 \%$ Brij-35, $0.1 \%$ polyethylene glycol 6000 for $4 \mathrm{~h}$ at $37^{\circ}$ C. Subsequently, MMP-1 was added to the phenol-red and serumfree DMEM culture supernatants from monocytes stimulated with SSc or HC serum, TLR8 or media only. The mixture of culture supernatant and activated human MMP-1 was then added to separate wells containing fluorogenic FS-6 substrate (500 $\mu \mathrm{M}$, Calbiochem). The effect of supernatant on the breakdown rate of FS- 6 by the activated MMP- 1 was visualised using a Perkin Elmer LS50b plate reader. For each sample, the rate of breakdown was compared with the standard containing MMP-1 $(6 \mu \mathrm{g} / \mathrm{ml})$, FS-6 $(50 \mu \mathrm{M})$ and phenol red-free DMEM (Gibco).

\section{Statistical analysis}

All data are presented as mean \pm SEM and differences between the groups were tested for their statistical significance by nonparametric two tailed $t$ test. A $p$ value $<0.05$ was considered statistically significant; $p$ values are expressed as follows: NS, significant; *0.05>p >0.01; **0.01 >p >0.001; *** $<<0.001$.

\section{RESULTS \\ TIMP-1 is increased in serum samples and overexpressed in circulating monocytes from patients with SSc}

TIMP-1 concentrations in serum of patients with SSc were raised compared with healthy individuals $(943 \pm 72 \mathrm{ng} / \mathrm{ml}$ vs 627 $\pm 70 \mathrm{ng} / \mathrm{ml}, \mathrm{p}=0.005$ ) (figure $1 \mathrm{~A}$ ), in keeping with a previously published study. ${ }^{24}$ TIMP-1 concentrations did not correlate with 

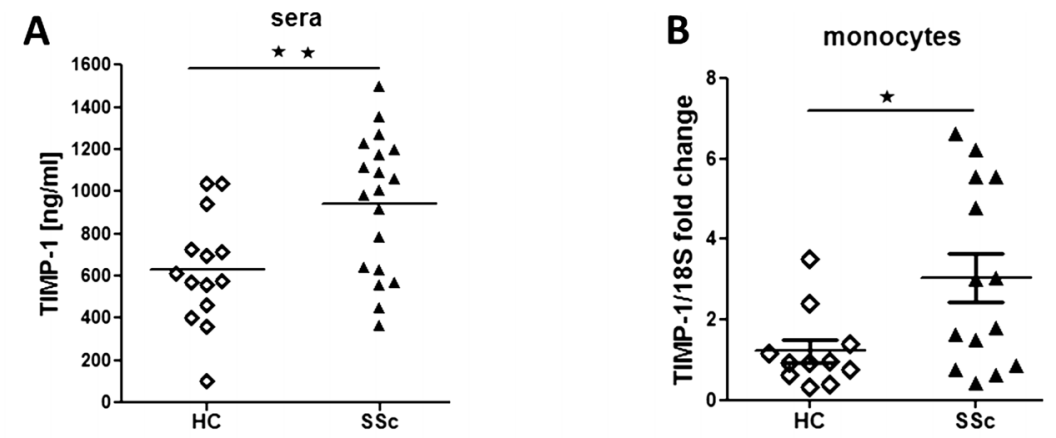

\section{C}
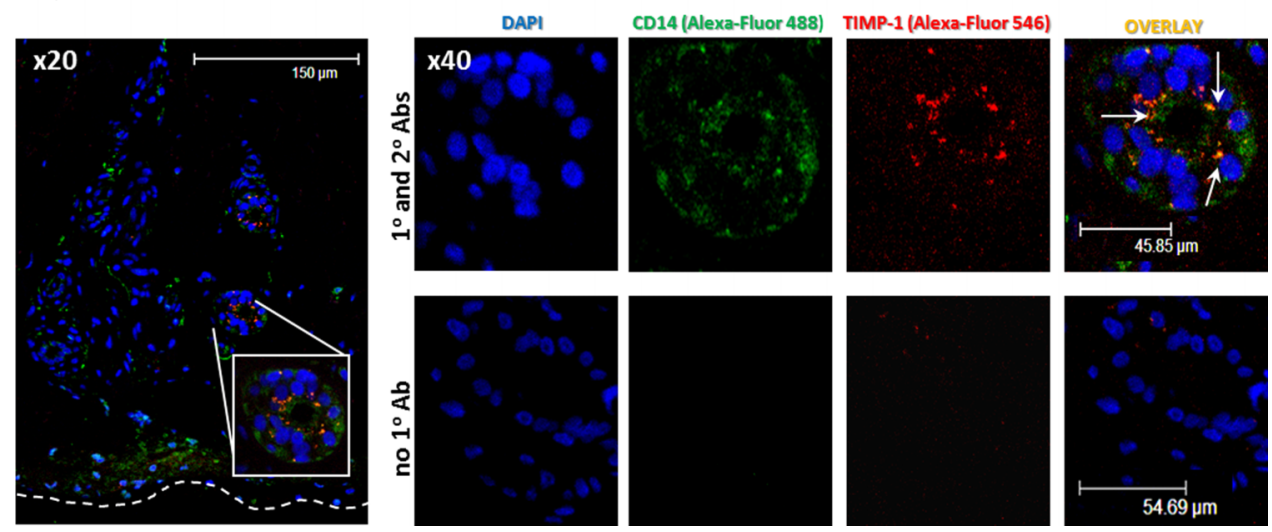

Figure 1 Tissue inhibitor of metalloproteinase-1 (TIMP-1) in serum samples and circulating monocytes. (A) TIMP-1 protein concentration was measured in serum samples of healthy controls $(H C)(n=14)$ or patients with systemic sclerosis $(S S c)(n=19)$. (B) Relative TIMP-1 expression in freshly isolated circulating CD14 monocytes from HC $(n=11)$ or patients with SSc $(n=14)$ was obtained. Relative expression levels of TIMP-1 were normalised to the $18 \mathrm{~S}$ housekeeping gene. Each value represents the mean; ${ }^{*} p<0.05,{ }^{*} p<0.01$. Paraffin-embedded SSc skin section. (C) The skin section was stained with CD14 and TIMP-1 primary and secondary antibodies (Abs; Alexa-Fluor488, Alexa-Fluor546, respectively) and further analysed by confocal microscopy (TCS SP2 UV LSM Leica Microsystems) using $\times 20$ (top side of the image represents the dermis and bottom the epidermis) and $\times 40$ magnification. White arrows indicate colocalisation between CD14 and TIMP-1.

skin thickening, disease onset, lung involvement, treatment status or autoantibody status (not shown). MMP-1 was below the detection limit of the assay. Furthermore, TIMP-1 gene expression in CD14 monocytes from patients with SSc and $\mathrm{HC}$ was analysed by qRT-PCR (figure 1B). Seven patients had diffuse cutaneous SSc, all positive for Scl-70 autoantibodies. The other patients had limited cutaneous disease and were positive for either anticentromere antibodies or antinuclear antibodies. The expression of TIMP-1 in SSc monocytes was significantly higher than in HC monocytes $(p=0.021)$. We also measured TIMP-2 and MMP-13 in serum samples, but we found no differences in serum concentrations (see online supplementary figure S1A,B).

\section{CD14 monocytes expressed TIMP-1 in the SSc skin}

To confirm that infiltrating CD14 monocytes contribute to skin fibrogenesis by TIMP-1 production, immunohistochemical staining of SSc skin was performed. It can be seen (figure 1C) that a paraffin-embedded skin section from a patient with early diffuse SSc expressed TIMP-1 by CD14 monocytes as indicated by overlaying images from confocal microscopy owing to colocalisation.

\section{Serum samples from patients with SSc induce de novo MyD88-dependent TIMP-1 production in HC monocytes}

Next, we investigated whether soluble factors present in SSc serum might contribute to the increased TIMP-1 or MMP-1 expression in circulating monocytes. To this end HC monocytes were cultured for $4 \mathrm{~h}$ with $10 \%$ serum samples from $\mathrm{HC}$, patients with SSc or patients with RA. As seen in figure 2A, $4 \mathrm{~h}$ after stimulation, gene expression of TIMP-1 in HC monocytes cultured in the presence of SSc serum (fold increase $2 \pm 0.2)$ was significantly raised compared with expression in HC $(p=0.01)$ or RA $(p=0.03)$ serum. Of note, TIMP-1 expression in monocytes was the highest after incubation with SSc serum samples containing Scl-70 autoantibodies, although this did not reach statistical significance. Interestingly, MMP-1 gene expression levels (figure $2 \mathrm{~B}$ ) after stimulation by either $\mathrm{HC}$ or SSc serum samples did not differ between the groups $(p=0.59)$. Similarly to the gene expression results, TIMP-1 protein level (figure 2C) was also significantly higher in monocytes cultured in SSc serum samples $(17 \pm 2 \mathrm{ng} / \mathrm{ml})$, serum samples from $\mathrm{HC}$ $(9 \pm 0.7 \mathrm{ng} / \mathrm{ml}, \mathrm{p}=0.008)$ or from patients with RA $(7 \pm 0.8$, $\mathrm{p}=0.003)$ and was highest in cultures with anti-Scl-70-positive serum samples $(24 \pm 7.7 \mathrm{ng} / \mathrm{ml})$ (not shown).

To confirm that TIMP-1 was produced de novo and not carried over via serum, HC monocytes were washed twice with cold phosphate-buffered saline, and TIMP-1 production was measured as previously (figure 2D). The level of TIMP-1 directly after stimulation with serum samples was similar to that of untreated cells in both monocytes stimulated with $\mathrm{HC}$ $(1.9 \pm 0.5 \mathrm{ng} / \mathrm{ml})$ and SSc serum samples $(1.9 \pm 0.3 \mathrm{ng} / \mathrm{ml})$, confirming the lack of remaining TIMP-1 after incubation of serum samples. Interestingly, $24 \mathrm{~h}$ later (figure 2D), the level of TIMP-1 increased in monocytes stimulated with SSc serum samples $(19 \pm 2.2 \mathrm{ng} / \mathrm{ml})$ compared with HC serum samples $(8 \pm 1.2 \mathrm{ng} / \mathrm{ml})$, but pretreatment with $50 \mu \mathrm{M}$ MyD88 inhibitory peptide 
A

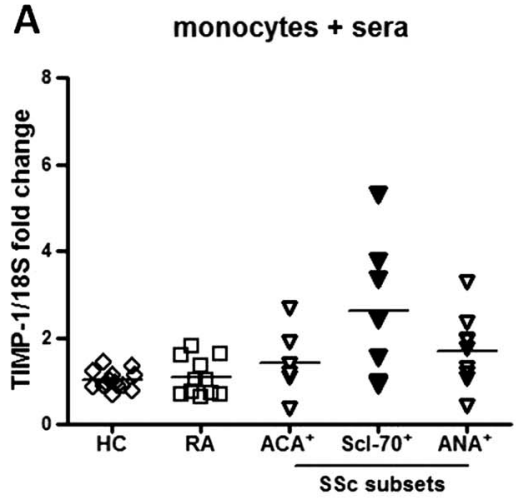

D

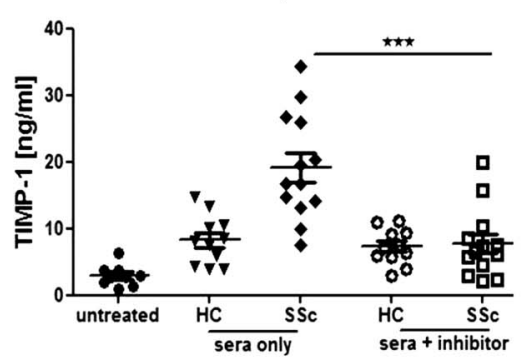

B

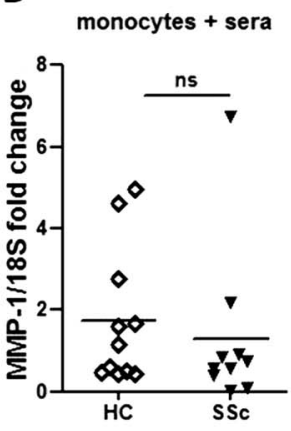

C
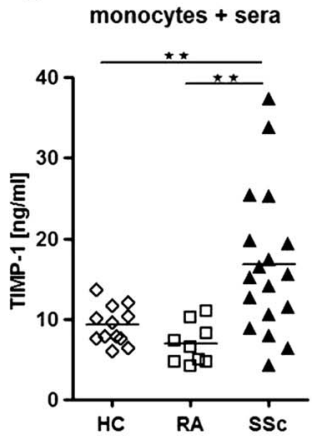

$E$

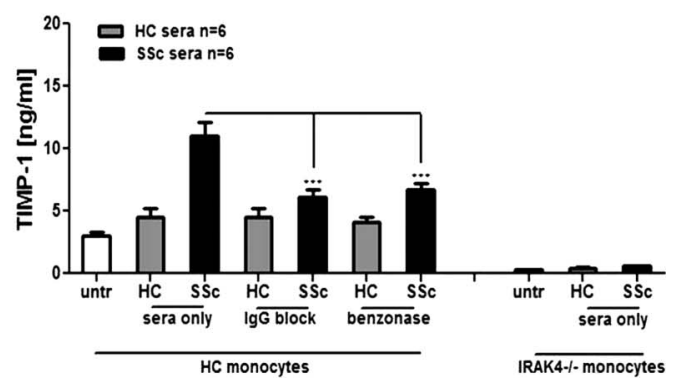

Figure 2 The effect of serum samples on the tissue inhibitor of metalloproteinase-1 (TIMP-1) and matrix metalloproteinase-1 (MMP-1) induction. (A) Healthy control $(\mathrm{HC})$ monocytes were treated for $4 \mathrm{~h}$ with serum samples from $\mathrm{HC}(\mathrm{n}=11)$, patients with rheumatoid arthritis $(\mathrm{RA})(\mathrm{n}=11)$ or patients with systemic sclerosis (SSc) defined by autoantibody positivity-anticentromere antibodies $(n=6), S c l-70(n=7)$, antinuclear antibodies $(\mathrm{n}=9)$ - and TIMP-1 gene expression was measured. (B) MMP-1 gene expression in HC monocytes cultured in SSc or HC serum was also analysed. Expression levels of TIMP-1 and MMP-1 were normalised to the $18 \mathrm{~S}$ housekeeping gene and each dot represents the mean. (C) HC monocytes were stimulated with HC, rheumatoid arthritis (RA) or SSc serum samples and TIMP-1 secretion was measured $24 \mathrm{~h}$ later. (D) HC monocytes were pretreated or not with MyD88 inhibitory peptide for $24 \mathrm{~h}$ and further stimulated over $4 \mathrm{~h}$ either with $10 \% \mathrm{HC}$ or SSc serum samples. De novo TIMP-1 secretion by HC monocytes was measured $24 \mathrm{~h}$ later. Results are representative of mean $\pm S E M$ of $H C(n=11)$ or $S S c(n=13)$ serum samples. (E) HC monocytes were pretreated with human IgG for $1 \mathrm{~h}$ before stimulation with HC or SSc serum samples (IgG block); HC or SSc serum samples were incubated with RNA/DNA endonuclease (benzonase) before sera stimulation; or IRAK4-/- monocytes were treated with HC and SSc serum samples and TIMP-1 secretion was measured. Statistical analysis showed that monocytes treated with SSc sera were comparable with monocytes of IgG blocked sera or monocytes stimulated with SSc sera treated with benzonase and ${ }^{*} p<0.05,{ }^{* *} p<0.01,{ }^{* *} p<0.001$. Data shown in A-E represent separate experiments. IRAK, IL-1R-associated kinase.

significantly attenuated de novo SSc sera-driven TIMP-1 secretion $(7.7 \pm 1.4 \mathrm{ng} / \mathrm{ml}, \mathrm{p}=0.0002)$. In contrast, preincubation with $50 \mu \mathrm{M}$ scramble peptide (figure $3 \mathrm{~A}, \mathrm{~B}$ ) did not affect TIMP-1 production, indicating TLR-dependent induction of TIMP-1 synthesis by SSc serum samples. Interestingly, the level of TIMP-1 slightly increased upon stimulation with HC serum samples (fold increase $=2.8$ ) compared with untreated cells, suggesting that other factors such as IL-10 or tumour necrosis factor $\alpha$ may also influence TIMP-1 production, but less effectively than SSc sera factors (fold increase $=6.6$ ). ${ }^{25}{ }^{2} 26$

Furthermore, incubation of HC monocytes with $4 \mu \mathrm{g} / \mathrm{ml}$ of human IgG for $1 \mathrm{~h}$ (in order to block Fc receptors (Fc $\gamma \mathrm{R})$ ) attenuated (fold decrease $=1.8$ ) TIMP-1 production upon sera activation (figure 2E). When SSc serum samples were treated with benzonase (to degrade DNA and RNA) before stimulation, TIMP-1 production was strongly inhibited (fold decrease $=1.8$ ). In contrast, IgG block or benzonase treatment of serum samples did not affect TIMP-1 induction by HC serum samples. This indicates that factors present in SSc sera stimulate TIMP-1 production via FcyR and/or nucleic acids. Furthermore, monocytes isolated from an IRAK4-deficient patient and stimulated with HC or SSc serum did not produce TIMP-1, demonstrating an important role of IRAK4 signalling pathway in sera-mediated TIMP-1 production.

\section{MMP activity is significantly reduced upon addition of supernatants from monocytes cultured in the presence of SSc serum}

To investigate whether the TIMP-1 secreted by monocytes is functionally active and impairs substrate breakdown by MMP we employed a functional matrix breakdown assay. FS-6 breakdown by MMP- 1 was significantly $(p=0.02)$ lower in the presence of supernatant from monocytes preincubated with SSc serum compared with unstimulated monocytes (figure 4). A robust reduction $(59 \pm 2 \%)$ in MMP-1 activity was seen when supernatant from SSc serum-stimulated monocytes was added to active MMP-1.

\section{SSc monocytes respond more vigorously to TLR agonists than HC monocytes as measured by TIMP-1 secretion}

To determine which TLR agonist drives TIMP-1 production, both HC and SSc monocytes were stimulated with a panel of TLR 1-9 agonists (figure 5A). Freshly isolated CD14 monocytes from $\mathrm{HC}$ and patients with SSc were treated with different TLRs or media alone for $24 \mathrm{~h}$, and the expression level of TIMP-1 was measured by ELISA. TLR4, TLR8 and TLR9 agonists, in particular, induced significantly strong TIMP-1 production in SSc monocytes compared with HC (TLR4 fold 


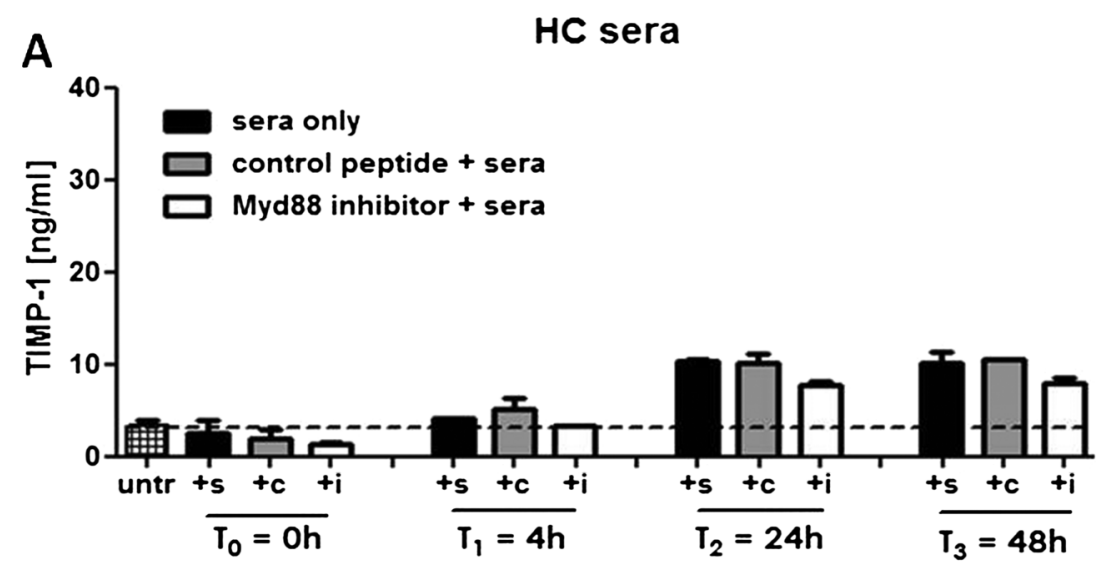

Figure 3 Kinetic analysis of the effect of serum samples on tissue inhibitor of metalloproteinase-1 (TIMP-1) induction. (A, B) healthy control (HC) monocytes were pretreated with MyD88 inhibitory peptide (white columns), control peptide (grey columns) or media only (black columns) for $24 \mathrm{~h}$ and further stimulated over $4 \mathrm{~h}$ either with $10 \% \mathrm{HC}$ or systemic sclerosis (SSc) serum samples. De novo TIMP-1 secretion by HC monocytes was measured directly after stimulation with sera (TO), $4 \mathrm{~h}$ (T1), $24 \mathrm{~h}$ (T2) or $48 \mathrm{~h}$ (T3) later. Results are representative of mean \pm SEM of two independent experiments for monocytes treated with $\mathrm{HC}$ or SSc serum samples.

increase $=4.0$, TLR9 fold increase $=3.8$, TLR8 fold increase $=3.4$ ). Pretreatment with MyD88 inhibitor reduced TIMP-1 production in TLR8-stimulated monocytes, but not when incubated with control peptide (figure 5C), confirming that TIMP-1 expression is MyD88-dependent after TLR activation. Peptide treatment did not affect cell viability as determined by MTS testing (data not shown). To extend our study, monocytes isolated from an IRAK4-deficient patient (deficiency confirmed by western blotting of fibroblasts; online supplementary figure S2) also did not secrete TIMP-1 after TLRs stimulation (figure 5A). In addition, TIMP-1 gene expression in monocytes from this patient was also lower compared with $\mathrm{HC}$ and SSc monocytes upon TLR8 agonist stimulation (figure $5 \mathrm{~B}$ ), confirming a role for TLR signalling in TIMP-1 production. Interestingly, TLR treatment did not induce TIMP-2 and only small quantities of MMP-13 after stimulation of TLR4, 8 and 9 (see online supplementary figure S1C,D).

\section{Supernatant from TLR8-stimulated SSc monocytes inhibits catalytic activity of MMP-1}

To confirm that TLR8 stimulation of monocytes inhibits matrix degradation we employed a functional matrix breakdown assay. Freshly isolated $\mathrm{HC}$ and SSc monocytes were cultured in phenolfree DMEM media and after $24 \mathrm{~h}$ stimulation with TLR8 agonist, supernatant was collected and added to preactivated
MMP-1. The percentage inhibition of FS-6 breakdown by supernatant from TLR8-treated SSc monocytes, normalised to untreated, was stronger (figure $6 \mathrm{~B}$ ) than that of $\mathrm{HC}$ monocytes (figure 6A). Statistical analysis of three independent experiments showed a similar pattern of significant TLR8-mediated MMP-1 inhibition by SSc monocytes (figure 6C) $(p<0.05)$.

\section{DISCUSSION}

The importance of the innate immune system and monocytes, in particular, in the pathogenesis of SSc is increasingly recognised. ${ }^{27-30}$ Traditionally, monocytes are seen as prototypic proinflammatory cells, but recent evidence has shown that their functional capabilities extend beyond that of cytokine production alone. In this study we explored the possibility that SSc monocytes may contribute to tissue fibrosis through preferential upregulation of TIMP-1 after stimulation with SSc serum samples or TLR agonists. This was based on the observation of increased TIMP-1 concentration in serum samples from patients with SSc and the recent identification of a subset of macrophages with a repair phenotype. ${ }^{8} 2431$ Another study found that in patients with SSc with interstitial lung disease, CD14 circulating monocytes in response to TLR4 agonist (lipopolysaccharide) overexpress CD163, IL-10 and CCL-18, which are known to stimulate collagen secretion by fibroblasts. ${ }^{30}$ We found that mRNA expression of TIMP-1 is higher in freshly 


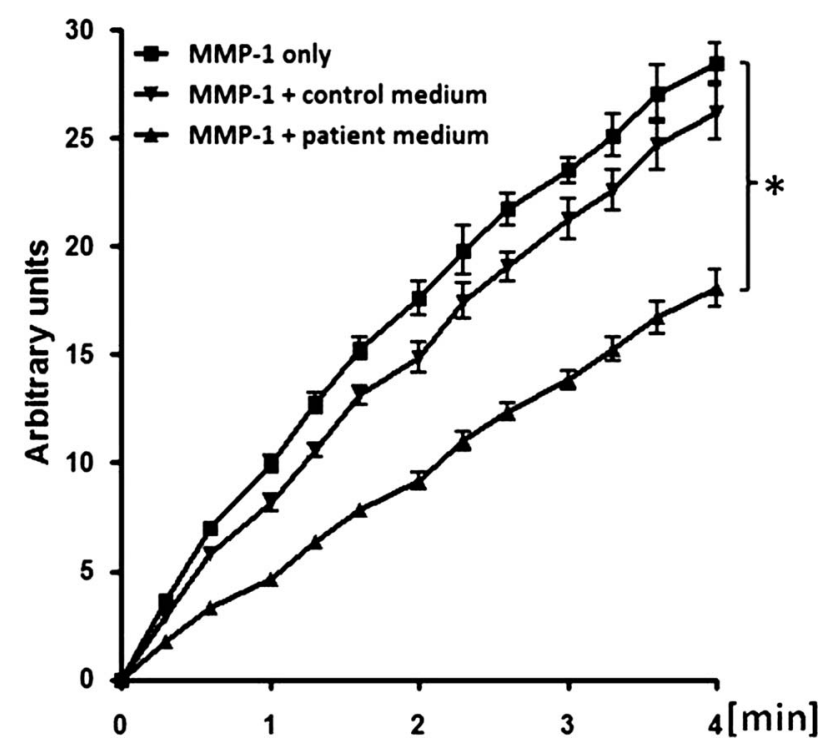

Figure 4 Functional matrix assay of monocytes stimulated with serum samples. Preactivated matrix metalloproteinases-1 (MMP-1) was added to the conditioned media of healthy controls (triangle) or systemic sclerosis (SSc) (circle) sera-treated or unstimulated monocytes (square) and fluorescent FS-6 breakdown product was measured over the time (min)]. Data shown represent the mean from duplicate values of one of three independent experiments ${ }^{*} p<0.05$ for comparison of the values of MMP-1 media only with MMP-1 SSc sera medium). isolated SSc monocytes than in HC monocytes. Also, serum from patients with SSc induces the expression and secretion of TIMP-1 but not MMP-1 in healthy monocytes. We observed TIMP-1 expression in CD14 cells in SSc skin (figure 1), raising the possibility that circulating monocytes expressing TIMP-1 migrate into skin to contribute to fibrogenesis. This is consistent with a recent gene expression study showing differential gene expression of MMP-1 and TIMP-1, with strongly increased TIMP-1 expression in SSc skin. ${ }^{17} 32$

Our results suggest that SSc serum samples are a source of agonists involved in TIMP-1 production, thus contributing to a profibrotic state. Likewise, Sacre et al ${ }^{33}$ showed that RA macrophages and synovial membrane cultures produce also a high level of tumour necrosis factor $\alpha$ and IL- 6 after TLR8 stimulation. Our results may imply that serum samples from patients with SSc contain ICs of autoantibodies that recognise selfnucleic acid-in particular, self-RNA or RNA-associated proteins. However, we did not detect any differences in RNA concentration or circulating ICs recognising C1q protein between $\mathrm{HC}$ and SSc serum samples (see online supplementary figure S3). This does not exclude the possibility that other ICs exist which contain RNA-associated autoantibodies that recognise other complement proteins. Such RNA-associated ICs might act as TLR8 ligands when they bind to abundantly expressed Fc $\gamma R$ on the surface of monocytes, leading to TIMP-1 production. Indeed, we showed that TIMP-1 induction was attenuated when Fc $\gamma$ R was blocked by human IgG and also when SSc serum samples were treated with DNA/RNA endonuclease

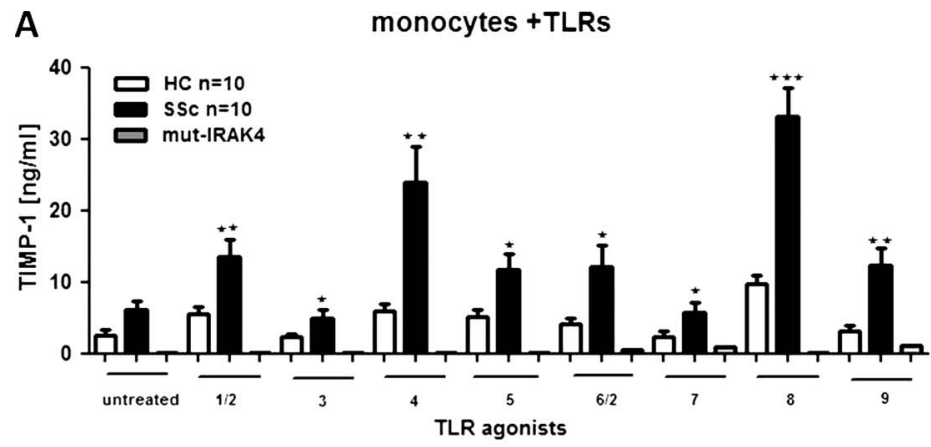

B

C
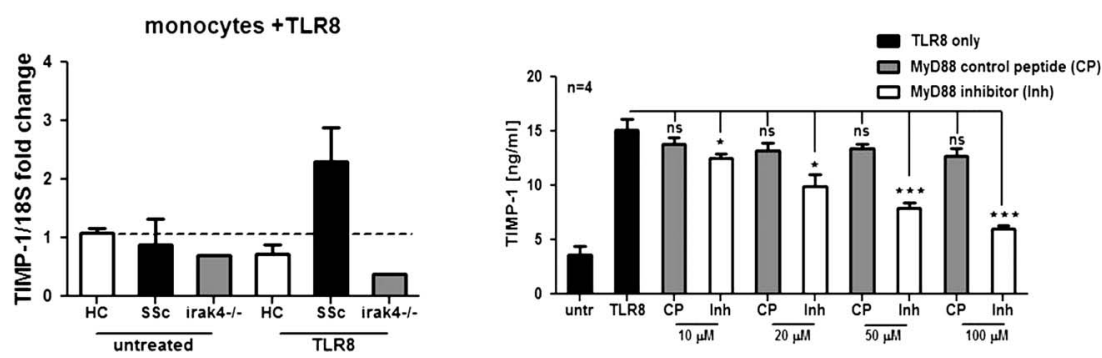

Figure 5 The effect of Toll-like receptor (TLR) agonists on tissue inhibitor of metalloproteinase-1 (TIMP-1) gene expression and protein secretion in monocytes. (A) Monocytes from healthy controls (HC) $(n=10)$ (white bars) or patients with SSc $(n=10)$ (black bars) and monocytes isolated from the mut-IRAK4 patient (grey bars) were incubated in the presence of different TLR agonists or media alone for $24 \mathrm{~h}$, and TIMP-1 secretion was determined. Statistical analysis showed that HC monocytes compared with SSc monocytes after individual TLR stimulation. (B) Relative TIMP-1 gene expression was determined and normalised to $18 \mathrm{~S}$ housekeeping gene of total RNA isolated from HC $(n=3)$ or SSc $(n=4)$ or mut-IRAK4 (grey bars) monocytes after $24 \mathrm{~h}$ stimulation with TLR8 agonist. (C) The effect of MyD88 inhibitor on TLR8-mediated TIMP-1 production. HC monocytes ( $\mathrm{n}=4$ ) were pretreated for $24 \mathrm{~h}$ with 10, 20, 50, $100 \mu \mathrm{M}$ MyD88 inhibitory peptide (white bars) or control peptide (grey bars) or media alone (black bars) and further stimulated with TLR8 agonist (ssRNA). Twenty-four hours later TIMP-1 production was measured. Statistical analysis showed a comparison of TLR8-stimulated monocytes compared with MyD88 inhibitor or control peptide treated monocytes, respectively. Each bar represents the mean \pm SEM, ${ }^{*} p<0.05,{ }^{* *} p<0.01,{ }^{* * *} p<0.001$. IRAK, IL-1R-associated kinase. 

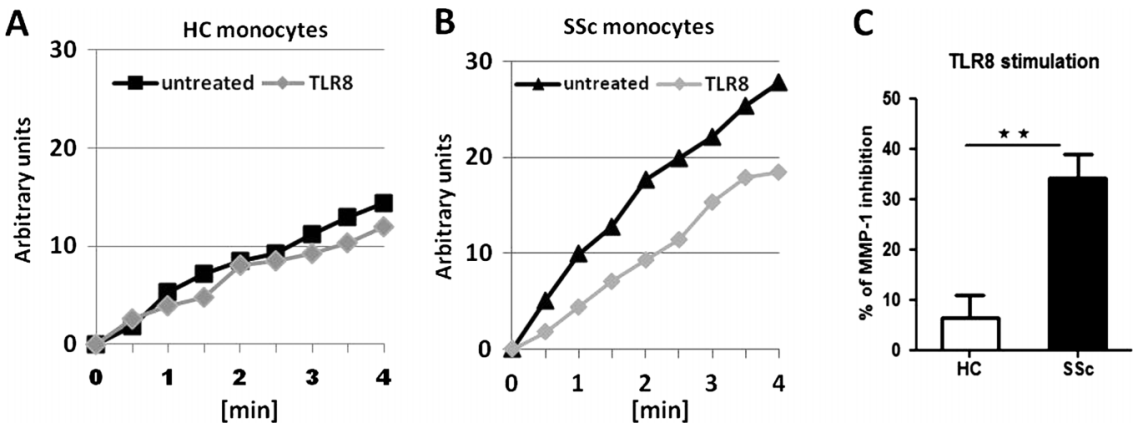

Figure 6 Functional matrix assay of Toll-like receptor 8 (TLR8)-stimulated monocytes. (A, B) Preactivated matrix metalloproteinases-1 (MMP-1) was added to the conditioned media of untreated (black line) or TLR8-simulated (grey line) healthy control (HC) or systemic sclerosis (SSc) monocytes and fluorescent FS-6 breakdown product was measured over time (min). (C) Statistical analysis of three independent experiments showing MMP-1 inhibition by $\mathrm{HC}$ and SSc monocytes pretreated with TLR8 agonist.

before stimulation. These findings strongly argue in favour of recognition of RNA by circulating ICs.

It was previously shown that opsonised apoptotic bodies carrying RNA-associated protein (Ro60) induce FcyR-dependent uptake. This IC uptake leads to TLR7/8 activation with subsequent release of proinflamatory and profibrotic factors by infiltrating macrophages in cardiac fibrosis, or pDCs in SSc. ${ }^{13} 2134$ Furthermore, the presence of ICs recognising self-RNA is responsible for disease manifestations found in patients and animal models of other autoimmune diseases, including of SLE and Sjögren's syndrome. ${ }^{35} 36$ Indeed, it was shown that serum samples from patients with SLE, containing autoantibodies to snRNPs that are taken up through the Fc $\gamma$ R, efficiently stimulate $\mathrm{pDCs}$ via TLR7/8 to secrete type I IFNs and, therefore, initiate SLE. ${ }^{32}$ Also, lupus-prone mice developed SLE, as a result of TLR7-dependent (mouse TLR8 is non-functional) activation of $B$ cells and pDCs. However, disease exacerbation was completely suppressed by the deletion of murine TLR7.

Notably, we also found that HC monocytes stimulated with patients' serum samples produce TIMP-1 in a MyD88-dependent fashion, as MyD88-specific inhibitor significantly attenuated TIMP-1 production. The same pattern of TIMP-1 reduction was seen in TLR8-stimulated HC monocytes in the presence of MyD88 inhibitor. In contrast, when HC monocytes were incubated with control peptide, TIMP-1 production was on a similar level to that of TLR8 activation only, confirming the specificity of the agonist. Monocytes isolated from a TLR-deficient patient also did not secrete TIMP-1 after stimulation with serum samples or TLR agonists, suggesting an important role of TLR signalling. The importance of our findings is underlined by recent evidence showing that MyD88-deficient mouse and rats were protected from fibrogenesis and pathogenic inflammation in a bleomycin-induced model of acute pulmonary injury indicated by reduced expression of collagen and TIMP-1. ${ }^{14}{ }^{3}{ }^{37}$ Furthermore, IRAK4 deficiency in humans abolished the induction of IFN $\alpha / \beta$ via TLR7-9 in blood cells. ${ }^{17}{ }^{18}$ Since MyD88 is also required for downstream signalling of IL-1 and IL-18, we measured the level of these cytokines in serum samples (data not shown). The concentration of IL-18 was increased in SSc serum samples, but direct IL-18 stimulation did not induce TIMP-1 production. Also, the concentration of IL-1 $\alpha$ and IL-1 $\beta$ did not differ between HC and SSc serum samples, suggesting that serum samples from patients with SSc contain factors which are MyD88-mediated but IL-1 and IL-18-independent in TIMP-1 expression.

Finally, this shift in balance towards TIMP-1 was functionally relevant as shown by the results from the matrix degradation assay. In particular, monocytes stimulated with SSc serum samples or TLR8 inhibited MMP-1 activity in comparison with untreated monocytes. These findings confirm the functional effect of TIMPs on MMP-1 activity. In this context it is of importance to note that (tissue-resident) macrophages have been shown to mediate wound healing responses. ${ }^{38}$ It is therefore tempting to speculate that circulating monocytes with profibrotic properties migrate to skin and internal organs to initiate and/or promote fibrosis in SSc.

In conclusion, our study shows a new link between TLR signalling and excessive TIMP-1 secretion in circulating monocytes from patients with SSc, supporting an important role for monocytes in the production of profibrotic factors. Therefore, treatments that downregulate TIMP-1 production may disrupt fibrogenesis in SSc and open up a new target.

Contributors MC, CAH: data collection, analysis, manuscript writing. TH, TS, AG, SO'R: data review. BG, TRDJR, SH: entry, data review. JMvL: project management, manuscript writing.

Funding This work was supported by the JGW Patterson Foundation.

Competing interests None.

Patient consent Obtained.

Ethics approval This study was approved by the research ethic committee (091/ H0905/11 for patients with SSC and 10/H0906/22 for the IRAK4-deficient patient). All patients gave informed consent.

Provenance and peer review Not commissioned; externally peer reviewed.

Data sharing statement Unpublished data are available upon request from the corresponding author.

Open Access This is an Open Access article distributed in accordance with the Creative Commons Attribution Non Commercial (CC BY-NC 3.0) license, which permits others to distribute, remix, adapt, build upon this work non-commercially, and license their derivative works on different terms, provided the original work is properly cited and the use is non-commercial. See: http://creativecommons.org/licenses/by-nc/3.0/

\section{REFERENCES}

1. Chizzolini C, Brembilla NC, Montanari E, et al. Fibrosis and immune dysregulation in systemic sclerosis. Autoimmun Rev 2011;10:276-81.

2. Yoshiji $\mathbf{H}$, Kuriyama $\mathrm{S}$, Miyamoto $\mathrm{Y}$, et al. Tissue inhibitor of metalloproteinases-1 promotes liver fibrosis development in a transgenic mouse model. Hepatology 2000;32:1248-54.

3. Visse $\mathbf{R}$, Nagase H. Matrix metalloproteinases and tissue inhibitors of metalloproteinases. Circ Res 2003;92:827-39.

4. Woessner JF. Matrix metalloproteinases and their inhibitors in connective tissue remodeling. FASEB J. 1991;5:2145-54.

5. Iredale JP, Benyon RC, Arthur MJ, et al. Tissue inhibitor of metalloproteinase-1 messenger RNA expression is enhanced relative to interstitial collagenase messenger RNA in experimental liver injury and fibrosis. Hepatology 1996;24:176-84.

6. Kikuchi K, Kubo M, Hoashi T, et al. Decreased MMP-9 activity in the serum of patients with diffuse cutaneous systemic sclerosis. Clin Exp Derm 2002;27:301-5. 
7. Kikuchi K, Kubo M, Sato S, et al. Serum tissue inhibitor of metalloproteinases in patients with systemic sclerosis. J Am Acad Derm 1995;33:973-8.

8. Montagnana M, Volpe A, Lippi G, et al. Relationship between matrix metalloproteinases/tissue inhibitors of matrix metalloproteinases systems and autoantibody patterns in systemic sclerosis. Clin Biochem 2007;40:837-42.

9. Singer AJ, Clark RAF. Cutaneous Wound Healing. New Engl J Med 1999:341:738-46.

10. Duan H, Fleming J, Pritchard DK, et al. Combined analysis of monocyte and lymphocyte messenger RNA expression with serum protein profiles in patients with scleroderma. Arthritis Rheum 2008;58:1465-74.

11. York MR, Nagai T, Mangini AJ, et al. A macrophage marker, siglec-1, is increased on circulating monocytes in patients with systemic sclerosis and induced by type i interferons and toll-like receptor agonists. Arthritis Rheum 2007:56:1010-20.

12. Seneviratne AN, Sivagurunathan B, Monaco C. Toll-like receptors and macrophage activation in atherosclerosis. Clin Chim Acta 2012;413:3-14.

13. Clancy RM, Alvarez D, Komissarova E, et al. Ro60-associated single-stranded RNA links inflammation with fetal cardiac fibrosis via ligation of TLRs: a novel pathway to autoimmune-associated heart block. J Immunol 2010;184:2148-55.

14. Couillin I, Vasseur V, Charron $\mathrm{S}$, et al. IL-1R1/MyD88 signaling is critical for elastase-induced lung inflammation and emphysema. J Immunol 2009;183:8195-202.

15. Singh MV, Swaminathan PD, Luczak ED, et al. MyD88 mediated inflammatory signaling leads to CaMKII oxidation, cardiac hypertrophy and death after myocardial infarction. J Mol Cell Card 2012;52:1135-44.

16. Dickie LJ, Church LD, Coulthard LR, et al. Vitamin D3 down-regulates intracellular Toll-like receptor 9 expression and Toll-like receptor 9-induced IL-6 production in human monocytes. Rheumatology 2010;49:1466-71.

17. Rajagopal D, Paturel C, Morel Y, et al. Plasmacytoid dendritic cellâ€"derived type I interferon is crucial for the adjuvant activity of Toll-like receptor 7 agonists. Blood 2010;115:1949-57.

18. Yang K, Puel A, Zhang S, et al. Human TLR-7-, -8-, and -9-mediated induction of IFN-alpha/beta and -lambda Is IRAK-4 dependent and redundant for protective immunity to viruses. Immunity 2005;23:465-78.

19. Eloranta ML, Lovgren T, Finke D, et al. Regulation of the interferon-alpha production induced by RNA-containing immune complexes in plasmacytoid dendritic cells. Arthritis Rheum 2009;60:2418-27.

20. Lovgren T, Eloranta ML, Kastner B, et al. Induction of interferon-alpha by immune complexes or liposomes containing systemic lupus erythematosus autoantigen- and Sjogren's syndrome autoantigen-associated RNA. Arthritis Rheum 2006;54:1917-27.

21. Kim D, Peck A, Santer D, et al. Induction of interferon-alpha by scleroderma sera containing autoantibodies to topoisomerase I: association of higher interferon-alpha activity with lung fibrosis. Arthritis Rheum 2008:58:2163-73.

22. Picard C, Casanova JL, Puel A. Infectious diseases in patients with IRAK-4, MyD88, NEMO, or IkappaBalpha deficiency. Clin Microbiol Rev 2011;24:490-7.

23. von Bernuth H, Picard C, Jin Z, et al. Pyogenic bacterial infections in humans with MyD88 deficiency. Science 2008:321:691-6.
24. Young-Min SA, Beeton C, Laughton R, et al. Serum TIMP-1, TIMP-2, and MMP-1 in patients with systemic sclerosis, primary Raynaud's phenomenon, and in normal controls. Ann Rheum Dis 2001:60:846-51.

25. Zhang Y, McCluskey K, Fujii K, et al. Differential regulation of monocyte matrix metalloproteinase and TIMP-1 production by TNF-alpha, granulocyte-macrophage CSF, and IL-1 beta through prostaglandin-dependent and -independent mechanisms J Immunol 1998;161:3071-6.

26. Lacraz S, Nicod LP, Chicheportiche R, et al. IL-10 inhibits metalloproteinase and stimulates TIMP-1 production in human mononuclear phagocytes. J Clin Invest 1995:96:2304-10

27. Tourkina $\mathbf{E}$, Bonner $\mathrm{M}$, Oates J, et al. Altered monocyte and fibrocyte phenotype and function in scleroderma interstitial lung disease: reversal by caveolin-1 scaffolding domain peptide. Fibrogenesis Tissue Repair 2011;4:15

28. Higashi-Kuwata N, Jinnin M, Makino T, et al. Characterization of monocyte/ macrophage subsets in the skin and peripheral blood derived from patients with systemic sclerosis. Arthritis Res Ther 2010;12:R128.

29. Ambarus CA, Krausz S, van Eijk M, et al. Systematic validation of specific phenotypic markers for in vitro polarized human macrophages. J Immunol Methods 2012:375:196-206.

30. Mathai SK, Gulati M, Peng X, et al. Circulating monocytes from systemic sclerosis patients with interstitial lung disease show an enhanced profibrotic phenotype. Lab Invest 2010;90:812-23.

31. Gorden KB, Gorski KS, Gibson SJ, et al. Synthetic TLR agonists reveal functional differences between human TLR7 and TLR8. J Immunol 2005;174: 1259-68.

32. Frost J, Ramsay MI, Mia R, et al. Differential gene expression of MMP-1, TIMP-1 and HGF in clinically involved and uninvolved skin in South Africans with SSc. Rheumatology 2012;51:1049-52.

33. Sacre SM, Lo A, Gregory B, et al. Inhibitors of TLR8 reduce TNF production from human rheumatoid synovial membrane cultures. J Immunol 2008;181: 8002-9.

34. Alvarez D, Briassouli P, Clancy RM, et al. A novel role of endothelin-1 in linking toll-like receptor 7-mediated inflammation to fibrosis in congenital heart block. J Biol Chem 2011:286:30444-54

35. Avalos AM, Busconi L, Marshak-Rothstein A. Regulation of autoreactive $B$ cell responses to endogenous TLR ligands. Autoimmunity 2010;43 $76-83$

36. Santiago-Raber M-L, Dunand-Sauthier I, Wu T, et al. Critical role of TLR7 in the acceleration of systemic lupus erythematosus in TLR9-deficient mice. J Autoimmun 2009;34:339-48.

37. Gasse $\mathbf{P}$, Mary C, Guenon I, et al. IL-1R1/MyD88 signaling and the inflammasome are essential in pulmonary inflammation and fibrosis in mice. $J$ Clin Invest 2007:117:3786-99.

38. Rodero MP, Khosrotehrani K. Skin wound healing modulation by macrophages. Int J Clin Exp Pathol 2010:3:643-53. 